\title{
FLORA DE IMPORTÂNCIA POLINÍFERA PARA Apis mellifera (L.) NA REGIÃO DE VIÇOSA, MG ${ }^{1}$
}

\author{
Anna Frida Hatsue Modro², Dejair Message ${ }^{3}$, Cynthia Fernandes Pinto da Luz e João Augusto Alves
} Meira Neto ${ }^{5}$

\begin{abstract}
RESUMO - Procurou-se conhecer a flora de importância polinífera para Apis mellifera (L.) na região de Viçosa, MG, em período de entressafra de mel, entre agosto e dezembro de 2005. O experimento foi realizado em dois apiários distintos, cada um com cinco colmeias. As cargas retidas nos coletores de pólen instalados nas colmeias foram analisadas quanto à origem botânica. As plantas em floração no entorno dos apiários foram coletadas e identificadas. A maioria das plantas de importância polinífera para abelhas na região de Viçosa era nativa, localizada em jardins e com hábito arbóreo. Pela análise palinológica, verificou-se que espécies como Anadenanthera colubrina, Arecaceae sp., Baccharis dracunculifolia, B. melastomaefolia, Coffea spp., Emilia sagittata, Eugenia uniflora, Mikania cordifolia, M. hirsutissima, Myrcia fallax, Psidium guajava, Vernonia condensata, V. diffusa, V. lanuginosa e V. mariana são potenciais recursos poliníferos a serem utilizados no período de entressafra do mel. Os resultados indicaram a importância de plantas localizadas em áreas abertas para o forrageamento de pólen por A. mellifera e confirmaram o potencial polinífero da região estudada, durante o período de entressafra do mel.
\end{abstract}

Palavras-chave: Abelha; Recurso proteico e Pólen apícola.

\section{FLORA OF POLLINIFEROUS IMPORTANCE FOR Apis mellifera (L.) IN THE REGION OF VIÇOSA, MG}

\begin{abstract}
The objective of this work was to study flora of polliniferous importance for Apis mellifera (L.) in the region of Viçosa-MG, during the period between honey harvests from August to December, 2005. The experiment was carried out in two different apiaries, each one with five beehives. The pellets retained in the pollen collectors in the beehives were analyzed with respect to their botanical origin. The flowering plants surrounding the apiaries were collected and identified. Most plants of polliniferous importance for the bees in the Viçosa region were native, located in gardens and with arboreal habitus. The palynological analysis showed that species such as Anadenanthera colubrina, Arecaceae sp., Baccharis dracunculifolia, B. melastomaefolia, Coffea spp., Emilia sagittata, Eugenia uniflora, Mikania cordifolia, M. hirsutissima, Myrcia fallax, Psidium guajava, Vernonia condensata, V. diffusa, $V$. lanuginose and V. mariana are potential polliniferous resources to be used during the period between honey harvests. The results indicate the importance of plants located in open areas for pollen scavenging by A. mellifera, confirming the polliniferous potential of the studied area during the period between honey harvests.
\end{abstract}

Keywords: Bee, Protein resource and Bee pollen.

\section{INTRODUÇÃO}

Na atividade apícola, a exploração comercial dos produtos das abelhas exige do apicultor, além do manejo adequado de suas colmeias, a ocorrência de recursos poliníferos e nectaríferos no entorno dos apiários.
As plantas, quanto à oferta de recursos, podem ser classificadas em três grupos: plantas nectaríferas, plantas poliníferas e plantas poliníferas-nectaríferas (VILLANUEVA, 2002; BARTH, 2005). Nesse sentido, a perspectiva brasileira para a apicultura é bastante

\footnotetext{
${ }^{1}$ Recebido em 05.10.2009 e aceito para publicação em 02.05.2011.

${ }^{2}$ Doutorado em Entomologia pela Escola Superior deAgricultura "Luiz de Queiroz", ESALQ/USP, Brasil. E-mail:<fridamodro@gmail.com>.

${ }^{3}$ Polo Regional de Desenvolvimento Tecnológico dos Agronegócios do Vale do Paraíba. E-mail: <dejair.message@gmail.com>.

${ }^{4}$ Instituto de Botânica, Centro de Pesquisa em Plantas Vasculares, Núcleo de Palinologia. E-mail: <cyluz@yahoo.com.br>.

${ }^{5}$ Departamento de Biologia Vegetal, Universidade Federal de Viçosa, UFV, Brasil. E-mail: <j.meira@ufv.br>.
} 
promissora, pois as características especiais de clima e a flora com potencial apícola não são fatores limitantes da produção (BARRETO et al., 2005). Apesar dos vários levantamentos da vegetação utilizada como recurso alimentar para abelhas realizados em diversas regiões do Brasil (BARTH, 2004), a Embrapa Meio-Norte (2003) afirmou que o conhecimento sobre a flora apícola do país ainda é insuficiente, tendo em vista a grande diversidade botânica encontrada em todo o território nacional.

Dados sobre as fontes de pólen disponíveis em determinada região são importantes no sentido de apresentar aos apicultores regionais alternativas de exploração da atividade apícola em períodos em que o mel não é o principal produto das abelhas; estabelecer períodos em que se faz necessário oferecer reforço proteico para as colmeias ou programar a implantação de culturas que possam disponibilizá-lo, direcionando projetos de recomposição vegetal, como o reflorestamento de áreas de conservação e implantação de corredores ecológicos. Dessa maneira, este estudo visou identificar as fontes de pólen utilizadas por abelhas africanizadas (Apis mellifera L.) na região de Viçosa, MG, no período de entressafra de mel através da análise palinológica de cargas de pólen.

\section{MATERIAL E MÉTODOS}

Esta pesquisa foi realizada na região de Viçosa, Estado de Minas Gerais, Brasil, abrangendo dois apiários localizados aproximadamente a $15 \mathrm{~km}$ de distância um do outro: Apiário UFV (2245’33,0"S latitude e 42`52’03,7"W longitude) e Apiário Mesmel (2049’04,9"S latitude e $42^{\circ} 54^{\prime} 33,7^{\prime \prime W}$ longitude). Essas regiões apresentam domínio de vegetação de Floresta Estacional Semidecidual (Mata Atlântica) com 60 anos de sucessão secundária, porém ainda com amplas áreas antropizadas, onde existem canteiros ornamentais com árvores e ervas (apiário UFV) e áreas de pastagem abandonada (apiário Mesmel).

Pelo sistema de Köppen, o clima da região é do tipo Cwb, tropical de altitude, com verões chuvosos (set.-nov.) e invernos frios e secos (abr.-set.), temperatura média anual oscilando entre 14 e $23^{\circ} \mathrm{C}$ e precipitação média anual de 1.403,8 mm (VALVERDE, 1958).

Em cada apiário experimental foram utilizadas cinco colmeias de abelhas africanizadas (Apis mellifera L.), cada qual com um coletor de pólen intermediário instalado em sua parte superior. As cargas de pólen foram retidas nos coletores durante o período de entressafra de mel, entre 12 de agosto e 13 de dezembro de 2005.

Tomando os dois apiários experimentais como ponto central, semanalmente percorreu-se uma área equivalente a um raio de cerca de 500-1.000 m, coletando as espécies vegetais em floração. As plantas foram identificadas, e a nomenclatura das espécies vegetais seguiu o sistema de Cronquist (1988).

As amostras quinzenais de pólen retidas nos coletores e nas anteras das plantas foram preparadas conforme o método padrão europeu de Maurizio e Louveaux (1965), sem aplicação de acetólise, adaptado para cargas de pólen por Almeida-Muradian et al. (2005). A identificação dos tipos polínicos foi baseada, principalmente, na coleção de referência de lâminas de microscopia com pólen das plantas floridas da região de estudo, assim como em catálogos especializados em morfologia polínica de espécies de diversas floras (SALGADO-LABOURIAU, 1973; MELHEM et al., 1984; ROUBIK; MORENO, 1991).

Para o cálculo das frequências do pólen, os tipos polínicos com média menor que $1 \%$ foram agrupados e denominados “Outros” e os com média maior que $1 \%$, avaliados individualmente.

\section{RESULTADOS}

Foram coletadas no entorno dos apiários 88 espécies de plantas, cujos tipos polínicos se assemelhavam com aqueles presentes nas cargas de pólen das abelhas (Tabelas 1 e 2). Dos 66 tipos polínicos identificados, apenas 19 apresentaram frequência maior que $1 \%$, pertencentes principalmente às famílias Asteraceae (5), Euphorbiaceae (2) e Myrtaceae (2) (Tabela 2; Figuras 1-20).

As famílias Asteraceae (21), Fabaceae (9), Myrtaceae (6) e Bignoniaceae (5) apresentaram maior diversidade de espécies botânicas com importância polinífera na região, no período de coleta, sendo as principais: Anadenanthera colubrina, Arecaceae sp., Baccharis dracunculifolia, B. melastomaefolia, Bidens pilosa, Citrus spp., Coffea spp., Cosmos caudatus, Emilia sagittata, Erigeron bonariensis, Eucalyptus spp., Eugenia uniflora, Eupatorium purpurascens, E. squalidum,

Revista Árvore, Viçosa-MG, v.35, n.5., p.1145-1153, 2011 
Tabela 1 - Plantas de importância polinífera para Apis mellifera (L.) na região de Viçosa, MG, entre agosto e dezembro de 2005 (Hábito: Arb = Arbusto; Arv = Arbóreo; Epi = Epífita; Herb = Herbáceo; Trep = Trepadeira; Hábitat: Bord = Borda de mata; Cult = Cultivada; Jard = Jardim; Mata = interior de mata; Past = Pasto sujo; Umid = Área úmida. Origem: Nat = Nativa; Oor = Outra origem. Local: U = Apiário UFV; e M = Apiário Mesmel).

Table 1 - Plants of polliniferous importance for Apis mellifera (L.) in the region of Viçosa-MG, from August to December 2005. (Habit: Arb = shrub; Arv = Arboreal; Epi = Epiphytic; Herb = Herbaceous; Trep = climbing plant. Habitat: Bord = forest edge; Cult=Cultivated; Jard = garden; Mata = woods; Past = pasture; Umid = Humid area. Origin: Nat = Native; Oor = Other origin. Local: $U=U F V$ apiary; and $M=$ Mesmel apiary).

\begin{tabular}{|c|c|c|c|c|c|c|}
\hline Família & Espécies & Nome Vulgar & Hábito & Habitat & Origem & Local \\
\hline \multirow{2}{*}{$\begin{array}{l}\text { Acanthaceae } \\
\text { Amaranthaceae }\end{array}$} & Justicia brandegeana Wassh. \& L. B. Sm.4,5 & Camarão-vermelho & Arb & Jard & Oor & $\mathrm{U}$ \\
\hline & $\begin{array}{l}\text { Alternanthera brasiliana (L.) } \\
\text { Kuntze }\end{array}$ & Carrapicho & Herb & Jard & Nat & $\mathrm{UM}$ \\
\hline Anacardiaceae & $\begin{array}{l}\text { Mangifera indica L. } .^{1,2,5} \\
\text { Spondias mombin L. }\end{array}$ & $\begin{array}{l}\text { Mangueira } \\
\text { Cajazeiro }\end{array}$ & $\begin{array}{l}\text { Arv } \\
\text { Arv }\end{array}$ & $\begin{array}{l}\text { Cult } \\
\text { Cult }\end{array}$ & $\begin{array}{l}\text { Oor } \\
\text { Nat }\end{array}$ & $\begin{array}{c}\mathrm{UM} \\
\mathrm{U}\end{array}$ \\
\hline Apiaceae & Petroselinum crispum (Mill.) Nyman ex A. W. Hill & Salsa & Herb & Cult & Oor & M \\
\hline Apocynaceae & Plumeria sp. & $(-)$ & Arb & Jard & $(-)$ & $\mathrm{U}$ \\
\hline Arecaceae & Arecaceae sp. ${ }^{5}$ & Palmeiras & Arv & Jard & $(-)$ & $\mathrm{U}$ \\
\hline \multirow[t]{21}{*}{ Asteraceae } & Asteraceae sp. & $(-)$ & Herb & Past & $(-)$ & $\mathrm{U}$ \\
\hline & Baccharis dracunculifolia DC. ${ }^{3,6}$ & Alecrim-vassoura & Arb & Past & Nat & M \\
\hline & Baccharis melastomaefolia Hook. \& Arn. & $(-)$ & Arb & Cult & Oor & M \\
\hline & Bidens pilosa L. ${ }^{1,2,3,5}$ & Picão-preto & Herb & Past & Nat & M \\
\hline & Cosmos caudatus Kunth & Cosmos & Herb & Jard & Oor & $\mathrm{U}$ \\
\hline & Emilia sagittata DC. & $(-)$ & Herb & Past & Nat & UM \\
\hline & Erigeron bonariensis L. & Buva & Arb & Past & Nat & M \\
\hline & Eupatorium purpurascens Shc. Bip. Ex Baker & $(-)$ & Trep & Past & Nat & M \\
\hline & Eupatorium squalidum DC. ${ }^{3}$ & Mata-pasto & Herb & Past & Nat & UM \\
\hline & Eupatorium sp. & $(-)$ & Herb & Past & $(-)$ & M \\
\hline & Helianthus debilis Nutt. & Girassol-de-jardim & Herb & Jard & Oor & $\mathrm{U}$ \\
\hline & Mikania cordifolia (L. f.) Willd. ${ }^{5}$ & Guaco & Trep & Bord & Nat & $\mathrm{U}$ \\
\hline & Mikania hirsutissima DC. & Cipó-cabeludo & Trep & Bord & Nat & M \\
\hline & Piptocarpha macropoda (DC.) Baker & $(-)$ & Arv & Bord & Nat & M \\
\hline & Sonchus oleraceus L. ${ }^{5}$ & Serralha & Herb & Past & Nat & M \\
\hline & Tagetes erecta $\mathrm{L}$. & Cravo-de-defunto & Herb & Jard & Oor & $\mathrm{U}$ \\
\hline & Vernonia condensata Baker & Boldo & Arb & Past & Oor & M \\
\hline & Vernonia diffusa Less. & Fumo-bravo & Arv & Bord & Nat & UM \\
\hline & Vernonia lanuginosa Gardner & $(-)$ & Herb & Past & Oor & M \\
\hline & Vernonia mariana Mart. ex Baker & Assa-peixe & Arb & Past & Nat & $\mathrm{M}$ \\
\hline & Wedelia paludosa DC., ${ }^{1,2}$ & Arnica-do-mato & Herb & Bord & Nat & $\mathrm{UM}$ \\
\hline Balsaminaceae & Impatiens walleriana Hook. f. & Beijo & Herb & Bord & Oor & $\mathrm{U}$ \\
\hline \multirow[t]{5}{*}{ Bignoniaceae } & Pyrostegia venusta (Ker Gawl.) Miers & Cipó-são-joão & Trep & Bord & Nat & M \\
\hline & Spathodea campanulata P. Beauv. ${ }^{2}$ & Espatódea & Arv & Jard & Oor & $\mathrm{U}$ \\
\hline & Stenolobium stans (L.) Seem. ${ }^{5}$ & Ipê-mirim & Arv & Jard & Nat & $\mathrm{U}$ \\
\hline & Tabebuia roseoalba (Ridl.) Sandwith & Ipê-branco & Arv & Jard & Nat & $\mathrm{U}$ \\
\hline & Tabebuia serratifolia (Vahl) G. Nicholson & Ipê-amarelo & Arv & Jard & Nat & M \\
\hline Boraginaceae & Cordia curassavica (Jacq.)Roem \& Schult. & Erva-urubu & Herb & Umid & Nat & M \\
\hline Brassicaceae & Brassica rapa $\mathrm{L}$ & Colza & Herb & Cult & Oor & $\mathrm{U}$ \\
\hline \multirow[t]{2}{*}{ Commelinaceae } & Gibasis schiedeana (Kunth) D. R. Hunt & Véu-de-noiva & Herb & Jard & Nat & $\mathrm{U}$ \\
\hline & Tradescantia zebrina Heynh. & Lambari & Herb & Jard & Oor & $\mathrm{U}$ \\
\hline Convolvulaceae & Ipomoea cairica (L.) Sweet. ${ }^{2,5}$ & Cipó-são-joão & Trep & Past & Nat & M \\
\hline Elaeocarpaceae & Muntingia calabura L. ${ }^{2}$ & Calabura & Arv & Cult & Oor & $\mathrm{U}$ \\
\hline \multirow[t]{2}{*}{ Euphorbiaceae } & Euphorbia pulcherrima Willd. Ex Klotzsch ${ }^{1,2,5}$ & Bico-de-papagaio & Arb & Jard & Oor & $\mathrm{U}$ \\
\hline & Joannesia princeps Vell. & Cutieira & Arv & Jard & Nat & $\mathrm{U}$ \\
\hline
\end{tabular}


Tabela 1 - Cont.

Table 1 - Cont

\begin{tabular}{|c|c|c|c|c|c|c|}
\hline \multirow[t]{9}{*}{ Fabaceae } & Anadenanthera colubrina (Vell.) Brenan ${ }^{4,5}$ & Angico & Arv & Mata & Nat & UM \\
\hline & Bauhinia variegata L. $^{2,5}$ & Pata-de-vaca & Arv & Jard & Oor & $\mathrm{U}$ \\
\hline & Caesalpinia peltophoroides Benth. ${ }^{1,2,4}$ & Sibipiruna & Arv & Jard & Nat & $\mathrm{U}$ \\
\hline & Calliandra brevipes Benth. ${ }^{2}$ & Esponja & Arb & Jard & Nat & $\mathrm{U}$ \\
\hline & Crotalaria sp. ${ }^{3}$ & $(-)$ & Arb & Jard & $(-)$ & $\mathrm{U}$ \\
\hline & Dalbergia nigra (Vell.) Allemao ex Benth. ${ }^{1,2}$ & Jacarandá-da-bahia & Arv & Past & Nat & M \\
\hline & Erythrina falcata Benth. & Corticeira & Arv & Jard & Nat & $\mathrm{U}$ \\
\hline & Faboideae sp. & $(-)$ & Herb & Jard & $(-)$ & M \\
\hline & Inga vera Willd. & Ingá & Arv & Mata & Nat & M \\
\hline Lacythidaceae & Lecythis pisonis Cambess. & Sapucaia & Arv & Jard & Nat & $\mathrm{U}$ \\
\hline \multirow[t]{2}{*}{ Lamiaceae } & Hyptis brevipes Poit. ${ }^{2,3}$ & Erva-canudo & Herb & Úmid & Nat & M \\
\hline & Leonurus sibiricus L. ${ }^{2}$ & Picão-branco & Herb & Cult & Oor & UM \\
\hline \multirow[t]{2}{*}{ Lauraceae } & Ocotea dispersa (Nees) Mez & $(-)$ & Arv & Mata & Nat & M \\
\hline & Persea americana Mill. ${ }^{5}$ & Abacateiro & Arv & Cult & Oor & M \\
\hline Liliaceae & Dracaena spp. & $(-)$ & Arb & Jard & $(-)$ & $\mathrm{U}$ \\
\hline Loranthaceae & Struthanthus concinnus Mart. $^{1}$ & Erva-de-passarinho & Trep & Mata & Nat & M \\
\hline Lythraceae & Cuphea sp. & Érica & Herb & Jard & Nat & $\mathrm{U}$ \\
\hline Magnoliaceae & Michelia champaca L. & Magnólia-amarela & Arv & Jard & Oor & $\mathrm{U}$ \\
\hline Malpighiaceae & Galphimia brasiliensis (L.) A. Juss. & Triális & Arb & Jard & Nat & $\mathrm{U}$ \\
\hline Malvaceae & Abutilon striatum Dicks. Ex Lindl. & Lanterna-chinesa & Arb & Jard & Oor & $\mathrm{U}$ \\
\hline \multirow{2}{*}{\multicolumn{2}{|c|}{$\begin{array}{l}\text { Melastomataceae Leandra aurea (Cham.) Cogn. } \\
\text { Tibouchina granulosa (Desr.) Cogn. }{ }^{5}\end{array}$}} & $(-)$ & Herb & Past & Nat & M \\
\hline & & Quaresmeira & Arv & Past & Nat & $\mathrm{UM}$ \\
\hline Meliaceae & Melia azedarach L. & Cinamomo & Arv & Jard & Oor & $\mathrm{U}$ \\
\hline \multirow[t]{6}{*}{ Myrtaceae } & Callistemon viminalis (Sol. Ex Gaertn.) G. Don & Calistemo & Arb & Jard & Oor & M \\
\hline & Callistemon sp. & $(-)$ & Arv & Jard & $(-)$ & $\mathrm{U}$ \\
\hline & Eugenia uniflora L. ${ }^{5}$ & Pitanga & Arb & Cult & Nat & $\mathrm{U}$ \\
\hline & Eucalyptus spp..$^{1,2,4,5,6}$ & Eucalipto & Arv & Cult & Oor & M \\
\hline & Myrcia fallax (Rich.) DC. & Guamirim-do-preto & Arv & Past & Nat & M \\
\hline & Psidium guajava L. ${ }^{2,4,5}$ & Goiabeira & Arv & Cult & Nat & $\mathrm{UM}$ \\
\hline Nyctaginaceae & Bougainvillea glabra Choisy & Primavera & Arb & Jard & Nat & $\mathrm{U}$ \\
\hline Polygalaceae & Diclidanthera elliptica Miers & $(-)$ & Trep & Mata & Nat & M \\
\hline Proteaceae & Grevillea robusta A. Cunn. ex R. Br. & $(-)$ & Arv & Jard & Oor & $\mathrm{U}$ \\
\hline Rosaceae & Rosa sp. ${ }^{1}$ & Rosa & Arb & Jard & Oor & $\mathrm{U}$ \\
\hline \multirow[t]{2}{*}{ Rubiaceae } & Borreria suaveolens G. Mey. & $(-)$ & Arv & Past & Nat & $\mathrm{UM}$ \\
\hline & Coffea spp. & Café & Arb & Cult & Oor & $\mathrm{UM}$ \\
\hline Rutaceae & Citrus spp. ${ }^{2,5}$ & Limão ou Laranja & Arb & Cult & Oor & $\mathrm{UM}$ \\
\hline Sapindaceae & Serjania lethalis A. St.-Hil. & Timbó & Trep & Bord & Nat & M \\
\hline Scrophulariaceae & Russelia equisetiformis Schltdl. \& Cham. & Russélia & Arb & Jard & Oor & $\mathrm{U}$ \\
\hline \multirow[t]{3}{*}{ Solanaceae } & Brunfelsia sp. & $(-)$ & Arb & Bord & Nat & M \\
\hline & Solanum granuloso-leprosum Dunal & Fumo-bravo & Arv & Bord & Nat & UM \\
\hline & Solanum velleum Roem. \& Schult. & $(-)$ & Herb & Past & Nat & M \\
\hline Sterculiaceae & Dombeya wallichii (Lindl.) K. Schum..$^{2,5}$ & Astrapéia & Arb & Bord & Oor & $\mathrm{U}$ \\
\hline Tropaeolaceae & Tropaeolum sp. & $(-)$ & Herb & Jard & Nat & $\mathrm{U}$ \\
\hline \multirow[t]{3}{*}{ Verbenaceae } & Lantana camara $\mathrm{L}^{3,5}$ & Camará & Herb & Past & Oor & UM \\
\hline & Petrea spp. & $(-)$ & Arb & Jard & Nat & UM \\
\hline & Verbenaceae sp. & $(-)$ & Arb & Past & $(-)$ & $\mathrm{M}$ \\
\hline
\end{tabular}

Plantas citadas como de importância apícola na Região Sudeste por: ${ }^{1}$ Iwama e Melhem (1979); ${ }^{2}$ Ramalho et al. (1990); ${ }^{3}$ Barreto (1999); ${ }^{4}$ Carvalho e Marchini (1999); ${ }^{5}$ Marchini et al. (2001); e ${ }^{6}$ Santana (2003).

Revista Árvore, Viçosa-MG, v.35, n.5., p.1145-1153, 2011 
Tabela 2 - Frequências mensais dos principais tipos polínicos (> 1\%) identificados em cargas de pólen coletadas por $A$. mellifera na região de Viçosa (MG), no período de entressafra do mel, entre agosto e dezembro de 2005.

Table 2 - Monthly frequencies of the major pollen types (>1\%) identified in pellets collected by A. mellifera in the region of Viçosa (MG), in periods between harvests from August to December 2005.

\begin{tabular}{|c|c|c|c|c|c|c|}
\hline \multirow{2}{*}{$\begin{array}{l}\text { Tipos polínicos } \\
\text { Famílias }\end{array}$} & & \multicolumn{5}{|c|}{ Meses (\%) } \\
\hline & & Ago & Set & Out & Nov & Dez \\
\hline Anacardiaceae & Anacardiaceae tipo 1 & 0,55 & 2,23 & 3,86 & 0,05 & 2,73 \\
\hline Arecaceae & Arecaceae tipo 1 & 1,25 & 2,99 & 1,27 & 7,23 & 13,68 \\
\hline \multirow[t]{5}{*}{ Asteraceae } & Baccharis & 11,27 & 29,25 & 0,04 & 0,01 & 0,45 \\
\hline & Elephantopus & 0,44 & 2,7 & 2,26 & 0,47 & 0,23 \\
\hline & Gochnatia & 10,78 & 1,48 & 0,02 & 0,02 & - \\
\hline & Senecio & 1,33 & 11,92 & 1,77 & 4,81 & 2,83 \\
\hline & Vernonia & 23,05 & 0,73 & 5,48 & 0,94 & - \\
\hline Bignoniaceae & Bignoniaceae tipo 1 & 0,07 & 0,13 & 0,28 & 0,01 & 9,07 \\
\hline Cecropiaceae & Cecropia & 31,41 & 19,13 & 1,62 & 6,01 & 1,97 \\
\hline \multirow[t]{2}{*}{ Euphorbiaceae } & Alchornea & - & - & 0,02 & 9,13 & - \\
\hline & Ricinus & 1,03 & 1,24 & 1,99 & 1,35 & 3,96 \\
\hline Fabaceae & Anadenanthera & - & 0,04 & 4,25 & 30,94 & 28,59 \\
\hline \multirow[t]{2}{*}{ Myrtaceae } & Eucalyptus & 0,55 & 6,51 & 8,52 & 4,96 & 4,1 \\
\hline & Myrcia & 9,85 & 6,42 & 14,39 & 3 & 12,4 \\
\hline Piperaceae & Piper & 0,07 & - & 9,64 & 0,14 & - \\
\hline Rubiaceae & Coffea & 1,4 & 3,35 & 19,05 & 18,05 & - \\
\hline Rutaceae & Citrus & 0,76 & 0,94 & 3,51 & 3,52 & 5,18 \\
\hline Scrophulariaceae & Scrophulariaceae tipo 1 & 3,32 & 0,51 & 4,28 & - & - \\
\hline Ulmaceae & Trema & 0,38 & 0,22 & 7,93 & 2,28 & - \\
\hline Outros & ------ & 2,49 & 10,21 & 9,82 & 7,08 & 14,81 \\
\hline
\end{tabular}

Eupatorium sp., Helianthus debilis, Mikania cordifolia, M. hirsutissima, Myrcia fallax, Psidium guajava, Pyrostegia venusta, Russelia equisetiformis, Sonchus oleraceus, Spondias mombin, Tagetes erecta, Vernonia condensata, V. diffusa, V. lanuginosa, V. mariana e Wedelia paludosa (Tabelas 1 e 2).

A maioria das plantas de importância polinífera para A. mellifera na região de Viçosa foi de origem nativa (62\%). O hábitat das plantas foi principalmente em jardins (40,9\%), seguido de pastagem abandonada (23,8\%), área de cultivo (14,8\%), beira de mata (12,5\%), mata (5,7\%) e área úmida (2,3\%). Quanto ao hábito vegetativo, a maioria foi arbóreo (34,1\%), seguido de herbáceo (29,5\%), arbustivo (27,3\%) e trepadeira (9,1\%) (Tabela 1).

Das plantas poliníferas, 44,32\% foram coletadas somente no apiário UFV e 37,5\% no apiário Mesmel, sendo $18,19 \%$ coletadas em ambos os apiários. Nas proximidades do apiário UFV, as plantas foram coletadas em jardim (79,49\%), área de cultivo (10,26\%), borda de mata (7,69\%) epasto sujo (2,56\%), com hábitos arbóreo (38,46\%), arbustivo (33,33\%), herbáceo $(25,64)$ e trepadeira $(2,56 \%)$. No apiário
Mesmel foram coletadas em pasto sujo (45,45\%), borda de mata (15,15\%), área cultivada (12,12\%), mata (12,12\%), jardim (9,09\%) e áreas úmidas (6,06\%), com hábito principalmente herbáceo (30,30\%), arbustivo (24,24\%), arbóreo (24,24\%) e trepadeira (21,21\%) (Tabela 1).

\section{DISCUSSÃO}

Os tipos polínicos identificados neste trabalho também foram mencionados como ocorrentes em Minas Gerais (BASTOS, 1995; BARTH, 2005) e no Rio de Janeiro (BARTH; LUZ, 1998; LUZ et al., 2007), sendo considerados, portanto, característicos da Região Sudeste do país (RAMALHO et al., 1991), o que confirma a influência das características botânicas regionais e as preferências alimentares inerentes de $A$. mellifera no seu comportamento de coleta de pólen.

Representantes das famílias encontradas neste trabalho foram coletados em outros levantamentos florísticos na região de Viçosa por Marangon et al. (2003), Meira-Neto e Martins (2003), Ribas et al. (2003) e Silva et al. (2004ab), indicando que essas famílias são frequentes na região e podem ser consideradas

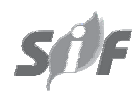

Revista Árvore, Viçosa-MG, v.35, n.5, p.1145-1153, 2011 

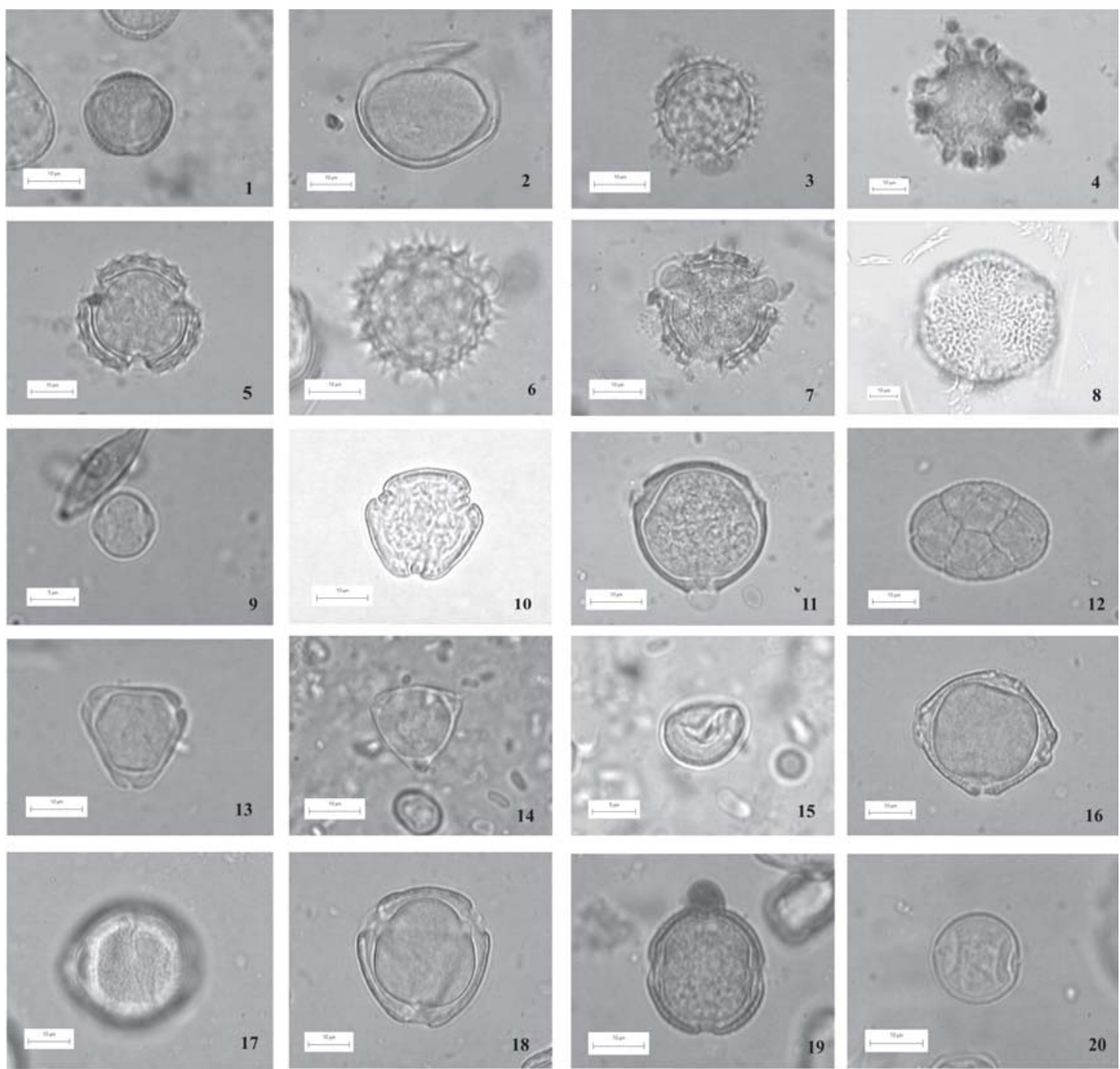

Figuras 1-20 - Fotomicrografias em microscopia óptica de grãos de pólen coletados por Apis mellifera L. na região de Viçosa, MG, no período de entressafra do mel, entre agosto e dezembro de 2005. Figura 1 - Anacardiaceae, tipo 1. Figura 2 - Arecaceae, tipo 1. Figura 3 - Asteraceae, tipo Baccharis. Figura 4 - Asteraceae, tipo Elephantopus. Figura 5 - Asteraceae, tipo Gochnatia. Figura 6 - Asteraceae, tipo Senecio. Figura 7 - Asteraceae, tipo Vernonia. Figura 8 - Bignoniaceae, tipo 1. Figura 9 - Cecropiaceae, tipo Cecropia. Figura 10 - Euphorbiaceae, tipo Alchornea. Figura 11 - Euphorbiaceae, tipo Ricinus. Figura 12 - Fabaceae, tipo Anadenanthera. Figura 13 - Myrtaceae, tipo Eucalyptus. Figura 14 - Myrtaceae, tipo Myrcia. Figura 15 - Piperaceae, tipo Piper. Figuras 16-18 - Rubiaceae, tipo Coffea. Figura 19 - Rutaceae, tipo Citrus. Figura 20 - Ulmaceae, tipo Trema (Escalas nas figuras $=10 \mu$, exceto nas Figuras 9 e $15=5 \mu$ ).

Figures 1-20 - Photomicrographies in optical microscopy of pollen grains collected by Apis mellifera L. in the region of Viçosa, MG, in the period between honey harvests from August to December 2005. Figure 1 - Anacardiaceae, type 1. Figure 2 - Arecaceae, type 1. Figure 3 - Asteraceae, type Baccharis. Figure 4 - Asteraceae, type Elephantopus. Figure 5 - Asteraceae, type Gochnatia. Figure 6 - Asteraceae, type Senecio. Figure 7 - Asteraceae, type Vernonia. Figure 8 - Bignoniaceae, type 1. Figure 9 - Cecropiaceae, type Cecropia. Figure 10 - Euphorbiaceae, type Alchornea. Figure 11 - Euphorbiaceae, type Ricinus. Figure 12 - Fabaceae, type Anadenanthera. Figure 13 - Myrtaceae, type Eucalyptus. Figure 14 - Myrtaceae, type Myrcia. Figure 15 - Piperaceae, type Piper. Figures 16-18 - Rubiaceae, type Coffea. Figure 19 - Rutaceae, type Citrus. Figure 20 - Ulmaceae, type Trema. (Figure scales $=10 \mu$, except in Figures 9 and 15 which was $5 \mu$ ).

Revista Árvore, Viçosa-MG, v.35, n.5., p.1145-1153, 2011 
como potenciais recursos poliníferos para A. mellifera, principalmente as famílias Asteraceae, Fabaceae, Myrtaceae e Bignoniaceae.

Assim como neste trabalho, muitas plantas da família Asteraceae são comumente observadas em canteiros ornamentais. Esses canteiros são conhecidos como manchas de recursos alimentares e têm a característica de possuir vários indivíduos da mesma espécie vegetal e com muitas flores por indivíduo, características que, segundo Struck (1994), Barth e Luz (1998) e Goulson (1999) são importantes na atração de insetos visitantes e polinizadores como A. mellifera.

Das plantas coletadas para este estudo, Vernonia diffusa e Anadenanthera colubrina foram as espécies indicadas para plantio em áreas em regeneração natural de pastagem e capoeira na região de Viçosa (PEREIRA, 2000). O levantamento das plantas que possivelmente contribuem com pólen para as abelhas pode ser utilizado em trabalhos de reflorestamento, para auxiliar na escolha de espécies a serem cultivadas nas proximidades de apiários, assim como para direcionar a atividade econômica apícola migratória para a produção de pólen.

De acordo com Ramalho et al. (1990), Cecropia pertence a um grupo de plantas heliófitas e anemófilas, e a presença desse tipo polínico comumente caracteriza a região de coleta como de borda de mata, mata ciliar ou floresta secundária da região neotropical. Moreti e Marchini (1998) observaram que as abelhas A. mellifera coletam recursos florais em uma altura de até $30 \mathrm{~m}$, o que foi confirmado neste estudo, pois a frequente coleta de Cecropia e a ocorrência de embaúbas em todo o fragmento secundário sugeriram que as abelhas coletam recursos poliníferos também localizados no dossel da mata.

Apesar de Spathodea campanulata ser ocorrente como planta polinífera para A. mellifera neste estudo e também ser citada por Ramalho et al. (1990) como polinífero para abelhas Trigonini (Tabela 1), esta espécie é conhecida por apresentar néctar tóxico para as abelhas (CINTRA et al., 2005), não sendo, portanto, recomendado seu uso em recomposição vegetal ou em áreas de jardinagem próximas a apiários.

Entre as principais plantas produtoras de pólen no período de entressafra (Tabelas 1 e 2), Baccharis dracunculifolia, Bidens pilosa e Eupatorium squalidum também foram recursos poliníferos coletados pelas abelhas em período de safra (abril a junho) na região de Viçosa, conforme levantamento realizado por Barreto (1999). Dessa maneira, essas plantas podem ser utilizadas como recurso polínico por um longo período do ano (abril a junho e agosto a dezembro); esse período torna-se ainda maior para a espécie $B$. pilosa, por ser mencionada por Santana (2003) como fornecedora de pólen também de dezembro a maio.

Plantas da família Arecaceae e as espécies Anadenanthera colubrina, Citrus spp., Eucalyptus spp., Eugenia uniflora, Mikania cordifolia, Psidium guajava e Sonchus oleraceus também foram mencionadas como plantas de importância para Apis mellifera na Região Sudeste do Brasil (MARCHINI et al., 2001; SANTANA, 2003).

A maior frequência de coletas de pólen por $A$. mellifera em plantas com hábitat em jardins, campos sujos e cultivadas em relação às plantas de mata também foi observada em Viçosa por Barth (2005), na Califórnia por Frankie et al. (2005) e no Rio de Janeiro por Luz et al. (2007). De acordo com Oliveira e Cunha (2005), no Brasil as abelhas africanizadas nidificam principalmente em áreas urbanas e formações vegetacionais abertas ou adulteradas, sendo dificilmente vistas ou coletadas no interior de florestas densas.

A predominância de plantas poliníferas localizadas em jardins nas proximidades do apiário UFV diferiu do ocorrido no apiário Mesmel, cujo hábitat mais frequente foi pasto sujo. Esse resultado reflete a flora dominante em cada localidade, confirmando a influência das características vegetativas do entorno dos apiários para a qualidade do produto apícola. De maneira semelhante, a predominância de plantas poliníferas de origem nativa possivelmente foi influenciada pela disponibilidade e qualidade de recursos no entorno dos apiários. Barth (2005) encontrou maior frequência de pólen de plantas exóticas em amostras de geleia real em colmeias localizadas próximo a canteiros ornamentais na região de Viçosa. O pólen observado na geleia real é proveniente da contaminação do pólen estocado dentro dos favos da colmeia, refletindo os recursos tróficos utilizados pelas abelhas em determinada região.

Revista Árvore, Viçosa-MG, v.35, n.5, p.1145-1153, 2011 


\section{CONCLUSÕES}

As famílias com maior diversidade de espécies botânicas com importância polinífera foram Asteraceae, Fabaceae, Myrtaceae e Bignoniaceae.

Houve preferência pelas abelhas por forragearem pólen em plantas nativas localizadas em áreas abertas e antropizadas.

A riqueza de plantas poliníferas em floração no período de entressafra de mel apresenta potencial para a produção comercial de pólen apícola heterofloral na região estudada. Destacando-se para esse fim as espécies Anadenanthera colubrina, Arecaceae sp., Baccharis dracunculifolia, B. melastomaefolia, Coffea spp., Emilia sagittata, Eugenia uniflora, Mikania cordifolia, $M$. hirsutissima, Myrcia fallax, Psidium guajava, Vernonia condensata, V. diffusa, V. lanuginosa e V. mariana.

\section{AGRADECIMENTOS}

À Ângela Maria da Silva Corrêa, do Instituto de Botânica (SP), pelo auxílio nas identificações polínicas e fotomicrografias.

Ao funcionário do Apiário Central da UFV Geraldo Neri Ferreira, pela competente contribuição no manejo das colmeias.

À CAPES, pela concessão de bolsa à primeira autora.

Ao Conselho Nacional de Desenvolvimento Científico e Tecnológico (CNPq), pela concessão de bolsa de produtividade (número 301220/2009-3) à terceira autora.

\section{REFERÊNCIAS}

ALMEIDA-MURADIAN, L. B. et al. Chemical composition and botanical evaluation of dried bee pollen pellets. Journal of Food Composition and Analysis, v.18, n.1, p.105-111, 2005.

BARRETO, L. M. R. C. Levantamento florístico e polínico e estudos melissopalinológico durante a principal safra da microrregião homogênea da zona da mata de Viçosa, MG. 1999. $74 \mathrm{f}$. Dissertação (Mestrado em Entomologia) -

Universidade Federal de Viçosa, Viçosa, MG, 1999.

BARRETO, L. M. R. C.; FUNARI, S. R. C.; ORSI, R. O. Pólen apícola: perfil da produção no Brasil. In: CONGRESSO DE APICULTURADEL MERCOSUR, 1., 2005, Punta Del Este. Anais... Punta Del Este: 2005. 20p.
BARTH, O. M.; LUZ, C. F. P.

Melissopalynological data obtained from a mangrove área near to Rio de Janeiro, Brazil. Journal of Apicultural Research, v.37, n.3, p.155-163, 1998.

BARTH, O. M. Melissopalynology in Brazil: a review of pollen analysis of honeys, propolis and pollen loads of bees. Scientia Agricola, v.61, n.3, p.342-350, 2004.

BARTH, O. M. Botanical resources used by Apis mellifera determined by pollen analysis of royal jelly in Minas Gerais, Brazil. Journal of Apicultural Research, v.44, n.2, p.78-81, 2005.

BASTOS, E. M. Espectro polínico do mel produzido em algumas áreas antrópicas de Minas Gerais. Revista Brasileira de Biologia, v.55, n.4, p.789-799, 1995.

CARVALHO, C. A. L.; MARCHINI, L. C. Plantas visitadas por Apis mellifera L. no vale do Paraguaçu, Município de Castro Alves, Bahia. Revista Brasileira de Botânica, v.22, n.2, p.333-338, 1999.

CINTRA, P.; MALASPINA, O.; BUENO, O. C. Plantas tóxicas para abelhas: artigo de revisão. Arquivos do Instituto Biológico, v.72, n.4, p.547-551, 2005.

CRONQUIST, A. The evolution and classification of flowering plants. New York: The New York Botanical Garden, 1988. 555p.

EMBRAPA MEIO-NORTE. Produção de mel. 2003. Disponível em: <http://

sistemasdeproducao.cnptia.embrapa.br/ FontesHTML/Mel/SPMel/importancia.htm>. Acesso em: 12 maio 2006.

FRANKIE, G. W. et al. Ecological patterns of Bees and their host ornamental flowers in two northern California cities. Journal of the Kansas Entomological Society, v.78, n.3, p.227-246, 2005.

GOULSON, D. Foraging strategies of insects for gathering nectar and pollen, and implications for plant ecology and evolution. Perspectives in Plant Ecology, Evolution and Systematics, v.2, n. 2, p.185-209, 1999. 
IWANMA, S.; MELHEM, T. S. The pollen spectrum of the honey of Tetragonisca angustula angustula Latreille (Apidae, Meliponinae). Apidologie, v.10, n.3, p.275-295, 1979.

LUZ, C. F. P.; THOMÉ, M. L.; BARTH, O. M. Recursos tróficos de Apis mellifera (Hymenoptera, Apidae) na região de Morro Azul do Tinguá, estado do Rio de Janeiro. Revista Brasileira de Botânica, v.30, v.1, p.27-34, 2007.

MARANGON, L. C.; SOARES, J. J.; FELICIANO, A. L. P. Florística arbórea da mata da pedreira, município de Viçosa, Minas Gerais. Revista Árvore, v.27, n.2, p.207-215, 2003.

MARCHINI, L. C. et al. Plantas visitadas por abelhas africanizadas em duas localidades do estado de São Paulo. Scientia Agricola, v.58, n.2, p.413-420, 2001.

MAURIZIO, A.; LOUVEAUX, J. Pollens de plantes melliferes dÉurope. Paris: U.G.A.F., 1965. 148p.

MEIRA-NETO, J. A. A.; MARTINS, F. R. Estrutura do sub-bosque herbáceo-arbustivo da mata da Silvicultura, uma floresta estacional semidecidual no município de Viçosa-MG. Revista Árvore, v.27, n.4, p.459-471, 2003.

MELHEM, T. S. et al. Planejamento para elaboração da "Flora polínica da Reserva do Parque Estadual das Fontes do Ipiranga (São Paulo, Brasil)”. Hoehnea, v.11, n.1, p.1-7, 1984.

MORETI, A. C. C. C.; MARCHINI, L. C. Altura de vôo das abelhas africanizadas (Apis mellifera L.) para coleta de alimentos. Scientia Agrícola, v.55, n.2, p.260-264, 1998.

OLIVEIRA, M. L.; CUNHA, J. A. Abelhas africanizadas Apis mellifera scutellata Lepeletier, 1836 (Hymenoptera: Apidae: Apinae) exploram recursos na floresta amazônica? Acta Amazônica, v.35, n.3, p.389-394, 2005.

PEREIRA, R. A. Mapeamento e caracterização de fragmentos de vegetação arbórea e alocação de áreas preferenciais para sua interligação no município de Viçosa, MG. 2000. 203f. Tese (Doutorado em Ciência Florestal) - Universidade Federal de Viçosa, Viçosa, MG, 2000.
RAMALHO, M.; KLEINERT-GIOVANNINI, A.; IMPERATRIZ-FONSECA, V. L. Important bee plants for stingless bees (Melipona and Trigonini) and Africanized honeybees (Apis mellifera) in neotropical habitats: a review. Apidologie, v.21, p.469-488, 1990.

RAMALHO, M. et al. Characterization of some southern Brazilian honey and bee plants through pollen analysis. Journal of Apicultural Research, v.30, n.2, p.81-86, 1991.

RIBAS, R. F. et al. Composição florística de dois trechos em diferentes etapas serais de uma floresta estacional semidecidual em Viçosa, Minas Gerais. Revista Árvore, v.27, n.6, p.821-830, 2003.

ROUBIK, D. W.; MORENO P. J. E. Pollen and spores of Barro Colorado Island. Monograph In Systematic Botany, v.36, p.1-268, 1991.

SALGADO-LABOURIAU, M. L. Contribuição à palinologia dos cerrados. Rio de janeiro: Academia Brasileira de Ciências, 1973. 291p.

SANTANA, A. G. Produção de própolis por Apis mellifera L. (africanizadas) e avaliação do uso do pólen na determinação de sua origem botânica. 2003. 48f. Dissertação (Mestrado em Entomologia) Universidade Federal de Viçosa, Viçosa, MG, 2003.

SILVA, N. R. S. et al. Composição florística e estrutura de uma floresta estacional semidecidual montana em Viçosa, MG. Revista Árvore, v.28, n.3, p.397-405, 2004a.

SILVA, C. T. et al.Avaliação temporal da florística arbórea de uma floresta secundária no município de Viçosa, Minas Gerais. Revista Árvore, v.28, n.3, p.429-441, 2004b.

STRUCK, M. Flowers and their insect visitors in the arid winter rainfall region of surthern África: observations on permanent plots. Insect visitation behaviour. Journal of Arid Environments, v.28, p.51-74, 1994.

VALVERDE, O. Estudo regional da Zona da Mata de Minas Gerais. Revista Brasileira de Geografia, v.20, n.1, p.1-82, 1958.

VILLANUEVA, G. R. Polliniferous plants aud foraging strategles Of Apis mellifera (Hymenoptera: Apidae) in the Yucatán Peninsula, Mexico. Revista de Biologia Tropical, v.50, n.3-4, p.1035-1044, 2002.

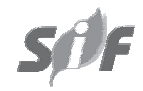

Revista Árvore, Viçosa-MG, v.35, n.5, p.1145-1153, 2011 
